\title{
A Frequency Adjustment Architecture for Energy Efficient Router
}

\author{
Wenliang $\mathrm{Fu}$, Tian Song \\ Beijing Laboratory of Intelligent Information Technology, School of Computer Science \\ Beijing Institute of Technology, Beijing, China, 100081 \\ \{fuwenl, songtian\}@bit.edu.cn
}

\begin{abstract}
With the rapid expansion of customer population and link bandwidth, energy expenditures of the Internet have been rising dramatically. To gain energy efficiency, we propose a novel router architecture, which allows each of its modules to adjust frequency according to traffic loads. Several modulation strategies are also discussed to ensure dwell time on low energy states and reduce blind switches. Our preliminary results show that the frequency adjustment router could save up to $40 \%$ of the total energy consumption.
\end{abstract}

\section{Categories and Subject Descriptors}

C.2.1 [Network Architecture and Design]: Network communications

\section{Keywords}

Energy Efficient Router, Frequency Adjustment

\section{INTRODUCTION}

Nowadays, much surplus and redundancy are introduced during network planning for rarely appeared traffic peak hours and device failures, wasting energy most of the time [1]. To cope with this situation, energy efficient technologies, such as smart port sleeping, dynamic buffer adapting and green routing, have been proposed to trade performance for energy efficiency in idle or low traffic time.

Smart port sleeping explores a sleep-wake mechanism between active and sleep modes for energy savings in the physical layer, and later leads to the establishment of standard IEEE 802.3az. However, due to considerable time and energy costs for transition, this method is only effective while port utilization rate is under $10 \%$ [2].

A. Vishwanath et al. explore buffer usage of routers and develop a buffer adapting algorithm for letting SRAM and DRAM buffers sleep while not being used [3]. Results show that up to $10 \%$ of the total energy consumption could be eliminated with negligible traffic congestion and latency.

From the network level, green routing exploits limiting traffic forwarding in a subset of network links. The essence of this idea is to optimize traffic modes for more energy efficient behaviors of network devices.

In this poster, we propose a frequency adjustment router architecture which allows each of its functional module to

Copyright is held by the author/owner(s). SIGCOMM'12, August 13-17, 2012, Helsinki, Finland. ACM 978-1-4503-1419-0/12/08.

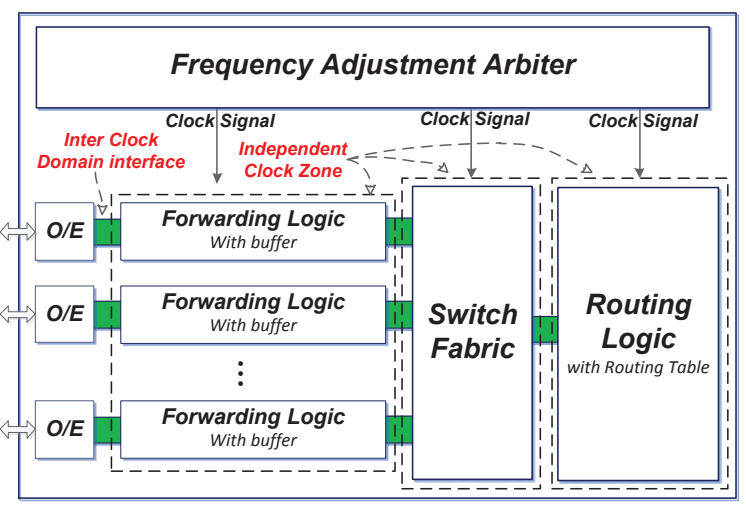

Figure 1: Block Diagram of Frequency Adjustment Router Architecture.

adopt different frequencies, offering various energy efficiencies, traffic capacities and processing latencies. Our frequency adjustment router has more potential for energy saving in the following aspects. First, aimed at functional modules which accounts for about $50 \%$ of a router's overall energy, our method has more energy saving potentials than other methods (ports and memory chips account for $10 \%$ and $11 \%$ of the total energy consumption respectively) [4]; Second, frequencies of each energy state could be customized for various traffic environments. Third, modulations between multiple frequencies are more flexible for strategic switching than simple sleep and active switching. In addition, our proposed frequency adjustment scheme is a essential supplement, not a replacement, for current energy efficient technologies, and the comprehensive utilization of those methods could certainly achieves higher energy efficiency.

\section{FREQUENCY ADJUSTMENT ROUTER ARCHITECTURE}

Our proposed architecture is shown in Fig.1. We treat functional modules as basic adjustment units, namely frequency adjustment zones. To support free frequencies modulation between modules, those units are connected with inter clock domain interfaces for handling asynchronous clock problems. Generally, the interface is an asynchronous FIFO based one-way data path, which collects signal sequences and stores them in FIFO according to one clock, and reads the signals from FIFO and replays them with the pace of another clock. In order to modulate frequencies of those units cooperatively, a frequency adjustment arbiter is introduced 

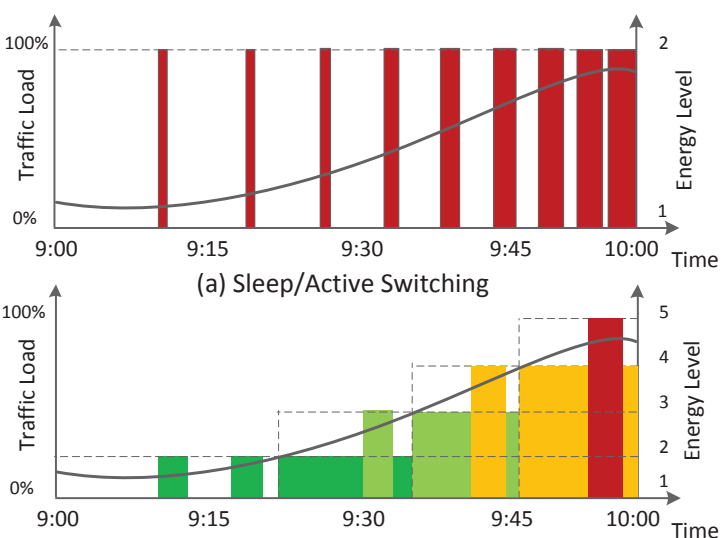

(c) Dual-boundary Switching

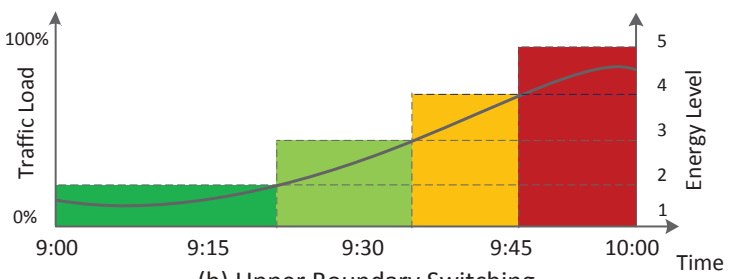

(b) Upper Boundary Switching

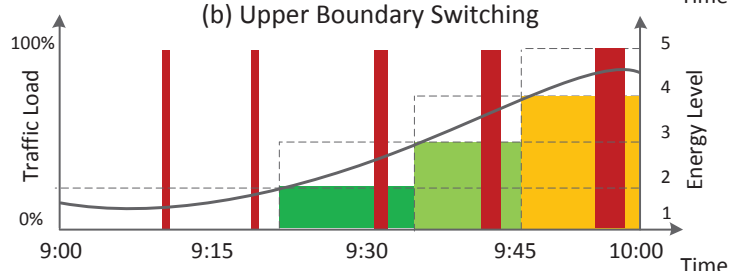

(d) Combined Switching

Figure 2: Energy State Switching Strategies.

to monitor system information, such as FIFO occupancy, port utilization and current energy status of each modules, and make frequency adjustment decisions.

\section{DYNAMIC FREQUENCY ADJUSTMENT STRATEGIES}

We developed several frequency switching strategies, distinguished by buffer occupancy threshold and cold down time between transitions, to guarantee stay time in low energy states and reduce blind switches, described as follow:

Sleep/active switching: Triggered by fixed buffer occupancy threshold, we propose a two-stage switch strategy as shown in Fig.2 (a), which allows modules to operate either in sleep mode for energy savings, or in active mode (marked as red) for processing packets in buffer.

Upper Boundary switching: This scheme permits multistage switching, and always adopts an upper frequency level that barely offering more capacity for handling the traffic. As shown in Fig.2 (b), energy states with different frequencies are distinguished by colors.

Dual-boundary switching: Fig.2 (c) describes a optimized strategy of upper boundary idea. By introducing a lower boundary, this scheme further reduces stay time in upper frequency levels with the cost of limited switches.

Combined switching: This strategy combines ideas of sleep/active switching and boundary switching, as shown in Fig.2 (d), treating low traffic time (such as nights) with sleeping strategy, and high traffic time with the aid of lower boundary for reducing frequent switches.

\section{EXPERIMENT AND RESULT}

We developed a router simulator which offers 10 frequencies range from $125 \mathrm{MHz}$ to $12.5 \mathrm{MHz}$, corresponding to $1000 \mathrm{Mbps}$ to $100 \mathrm{Mbps}$ respectively in traffic capacity. The experiments were based on real traces captured bidirectionally from a office network of 1000 users, spinning over a period of more than three hours and the loss rate was kept under 1\%. The preliminary results are shown in Fig.3, by limiting switches with preset cold down time between transitions and processing packets in buffer at the maximum speed, the combined strategy achieved the most significant savings than other methods.

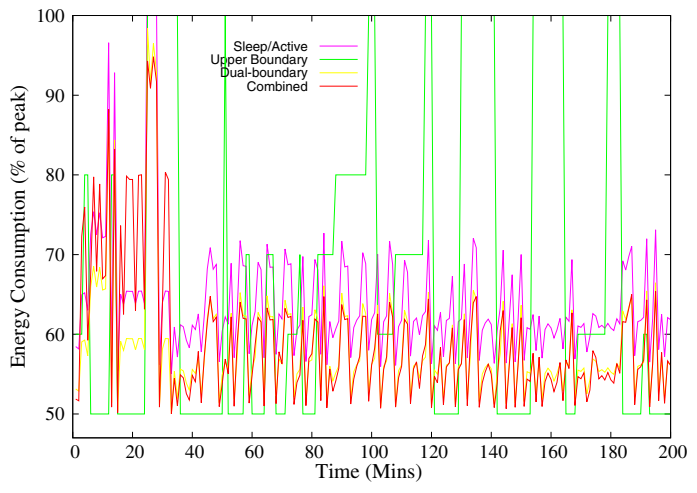

Figure 3: Frequency Adjustment for Energy Efficiency.

\section{CONCLUSION}

In this poster, we proposed a novel frequency adjustment router architecture, which allows each of its modules to adopt multiple and different frequencies. In addition, several frequency adjustment strategies are also described for tuning various network environments. Simulations on real traces show that our frequency adjustment router could save up to $40 \%$ of the total energy consumption.

\section{REFERENCES}

[1] The Applied Research Group. http://ipmon.sprint.com/.

[2] P. Reviriego, K. Christensen, J. Rabanillo, and J. Maestro. An initial evaluation of energy efficient ethernet. Communications Letters, IEEE, (99):1-3, 2011.

[3] A. Vishwanath, V. Sivaraman, Z. Zhao, C. Russell, and M. Thottan. Adapting router buffers for energy efficiency. In Proceedings of the Seventh COnference on emerging Networking EXperiments and Technologies, page 19. ACM, 2011.

[4] R.S. Tucker, R. Parthiban, J. Baliga, K. Hinton, R.W.A. Ayre, and W.V. Sorin. Evolution of wdm optical ip networks: A cost and energy perspective. Journal of Lightwave Technology, 27(3):243-252, 2009. 